\title{
Quelques petites règles (et suggestions) de l'auteur des Règles de la méthode sociologique
}

Small rules (and suggestions) of the author of the Règles de la méthode sociologique

Jean-François Bert

\section{OpenEdition}

\section{Journals}

Édition électronique

URL : http://journals.openedition.org/ress/3896

DOI : $10.4000 /$ ress.3896

ISBN : 1663-4446

ISSN : 1663-4446

Éditeur

Librairie Droz

Édition imprimée

Date de publication : 15 décembre 2017

Pagination : 41-56

ISSN : 0048-8046

\section{Référence électronique}

Jean-François Bert, «Quelques petites règles (et suggestions) de l'auteur des Règles de la méthode sociologique », Revue européenne des sciences sociales [En ligne], 55-2 | 2017, mis en ligne le 15 décembre 2020, consulté le 05 janvier 2021. URL : http://journals.openedition.org/ress/3896 ; DOI https://doi.org/10.4000/ress.3896 


\title{
QUELQUES PETITES RÈGLES (ET SUGGESTIONS) DE L'AUTEUR DES RĖGLES DE LA MÉTHODE SOCIOLOGIQUE
}

JEAN-FRANÇOIS BERT

Université de Lausanne Jean-Francois.Bert@unil.ch

\begin{abstract}
Résumé. Une nouvelle lecture de l'œuvre d'Émile Durkheim? Sans prétendre en arriver là, le présent article vise à contextualiser davantage les travaux du sociologue français et à en corriger certaines interprétations accoutumées, à l'aide de l'anthropologie des savoirs et de l'histoire des pratiques savantes. L'enjeu est ici de descendre d'un niveau pour essayer de faire apparaître ce qui semble important aux yeux de Durkheim lorsqu'il écrit et pratique sa sociologie, à savoir les questions de style, d'exposition ou encore d'argumentation.
\end{abstract}

Mots-clés: argumentation, correspondance, écriture, Émile Durkheim, Henri Hubert, Marcel Mauss, pratiques savantes, style.

\begin{abstract}
Without intending to promote a new reading of Émile Durkheim's work, this article simply aims at contextualizing further the French sociologist's works so as to revise some of their usual interpretations through the contribution of the anthropology of knowledge and the history of scholarly practices. What is at stake here is the necessity to go deeper into the text in order to bring to light what Durkheim assumed to be important as he was writing and practicing his sociology, namely issues of style, exposition as well argumentation.
\end{abstract}

Keywords: argumentation, Émile Durkheim, Henri Hubert, letters, Marcel Mauss, scholarly practices, style, writing. 
«Rattacher les idées, les tendances sociales à un état social défini, ne pas les laisser en l'air, mais montrer qu'elles font partie du réel, qu'elles en viennent et y retournent, l'expriment et le modifient, tout cela est très neuf.»

Durkheim, I899b, p. 430-431

Il ne fait aucun doute qu'il y a dans l'œuvre d'Émile Durkheim une ambition théorique et méthodologique proclamée. Tout en traitant de problèmes qu’il désigne comme «spéciaux», par rapport aux «généralités » de l’ancienne philosophie sociale (Durkheim, 1915, p. I3), qu'il s'agisse du mariage, de la famille, de la religion ou du suicide, Durkheim exprime son goût pour les questions de méthode et épistémologiques, et, plus largement, ce qui touche à la théorie de la connaissance. Cependant, on doit aussi faire l'hypothèse que, comme enseignant, chargé de cours de «Science sociale et pédagogie» à Bordeaux à partir de i887, puis titularisé comme professeur de «Science sociale» et enfin, à compter de 1902, de «Science de l'éducation» et de «Science de l'éducation et sociologie » à la Sorbonne, il trouva des manières plus «simples» d'exprimer, devant ses étudiants, les différents enjeux de sa réflexion; remanier certaines de ses propositions, et même donner corps à des idées souvent abstraites ${ }^{1}$. En un mot, utiliser des «ficelles», des «trucs», pour parler comme Howard Becker, qui sont ces «règles de bon sens tirées de l'expérience, comme celle qui veut qu'en mettant de jolis timbres de collection sur vos enveloppes de réponse, vous inciterez davantage de personnes à renvoyer leur questionnaire. » (Becker, 2002 [1988], p. 23-24).

I C'est en tout cas ce que nous laisse entendre cette remarque: «Quand nous étions professeur de lycée, il nous est arrivé bien souvent de demander aux élèves des classes de mathématiques quelle idée ils se faisaient du nombre, de la grandeur, de la quantité, du raisonnement des limites, etc., et de constater, par l'absence de toute réponse, qu'ils ne s'étaient jamais et qu'on ne leur avait jamais posé ces questions. Ils maniaient donc tous les jours ces idées et ces manières de raisonner, machinalement et sans comprendre le sens et la portée [...]. Ce qui concerne les méthodes scientifiques n'est traité qu'ensuite et comme une simple application des principes qui ont été antérieurement posés. On ne les observe donc pas, à parler exactement, mais on ne s'en occupe que pour prescrire ce qu'elles doivent être.» (Durkheim, 1895a, p. 425). 
La lecture que nous proposons ici de certains textes de Durkheim se situe à mille lieues de l'histoire des théories et des méthodes, de l'histoire des disciplines et de leur institutionnalisation, de la sociologie des controverses ou encore de l'analyse des modalités de la réception, hier et aujourd'hui, d'un auteur devenu un classique. Alors que ces perspectives fixent leur attention sur les résultats et les contenus formalisés dans des écrits achevés, nous voulons jeter un regard différent sur la dimension pratique du travail savant de Durkheim. Plus particulièrement, rendre visible ce qui est habituellement dissimulé dans le processus de la recherche en posant d’autres questions: comment, après avoir choisi un sujet d'étude, Durkheim décide-t-il de planifier son travail? Comment le fait-il tout en continuant à se laisser surprendre par une découverte, une suggestion ou une idée nouvelle? Quelles sont ses habitudes graphiques, ses pratiques de lecture et d'écriture, les routines qui structurent sa journée? Par chance, ses textes fourmillent de petites remarques qui sont pour lui autant d'occasions d'affirmer les principes de base de son activité de sociologue et d'une expérience de recherche progressivement consolidée.

Cette démarche s'inscrit dans le cadre d'une anthropologie du travail intellectuel ou d'une histoire matérielle des pratiques savantes qui est de plus en plus revendiquée comme un moyen efficace de contextualiser les productions écrites et, en même temps, d'en désencombrer la lecture et donc l'interprétation (Fabre, 1997; Jacob, 20I1 ; Bert, 20I2; Topalov, 20I2; Bacciochi et Mariot, 2015). Démarche qui tient, sans doute aussi, de ce que Durkheim avançait déjà pour expliquer les fonctions mentales supérieures - concernant notamment le raisonnement - en cherchant à les mettre en rapport avec les conditions sociales et les contraintes matérielles qui déterminent des manières particulières d'organiser la pensée (Durkheim, I899a, p. 5I). 


\section{LA VÉRITÉ SCIENTIFIQUE OU L’EFFICACITÉ DIDACTIQUE? LE DILEMME DE L'ENSEIGNANT}

Le Durkheim pédagogue qui doit trouver des ficelles pour exprimer devant les apprentis sociologues le cœur de sa méthode affleure dans les archives de ses étudiants, et particulièrement dans celles de Marcel Mauss qui à Bordeaux, entre 1890 et 1895 , prend note des enseignements de son oncle ainsi que des discussions informelles animées par lui. C'est le cas d'une «observation» de Durkheim sur la morale kantienne et l'intérêt qu'elle présente pour la sociologie. Si la loi morale, comme l'assure Durkheim que reprend verbatim Mauss, est pour Kant une métaphore de la puissance coercitive de la société, encore faut-il « définir la morale, choisir entre la morale objective et la morale subjective. Il fallait définir aussi le fonctionnement économique. Ensemble d'institutions dont la fonction serait de produire, et de répartir, pour la consommation [...]. De ce côté là on verrait des différences un peu plus déterminées, des faits nouveaux »².

C'est durant ce moment bordelais que Mauss constitue son bagage théorique, se familiarise avec des procédés de travail et acquiert la maîtrise des opérations qui lui seront indispensables par la suite: des plus concrètes (lire un livre ou faire un fichier) aux plus abstraites (aborder un problème, exposer sa pensée selon un ordre réfléchi). C'est d’ailleurs cette maîtrise qui inquiète le plus Durkheim, perplexe du style pour le moins singulier de son neveu qu'il trouve sur trop de points éloigné des attendus de l'épreuve de l'agrégation en philosophie . C'est en marge des dissertations préparatoires de Mauss qu'il exprimera ses remarques, comme dans ce commentaire qu'il griffonne après sa lecture de la dissertation «De la valeur scientifique des classifications ». Si les félicitations sont de rigueur («bonne dissertation», «effort louable», etc.), Durkheim souligne très vite la limiteprincipale: «Les questions ne sont pas

2 «Obs. de Durkheim», sans date, Fonds Mauss, Collège de France, 57 CDF 6-I-2.

3 Durkheim est au jury de l'agrégation. Un élément biographique important que Mauss rappelle: «L'activité professorale de Durkheim fut considérable et les sujets de son enseignement furent toujours renouvelés. Dès I89|, il fut du jury d'agrégation - mais déjà, depuis I888, il ne manqua jamais, pour ses élèves candidats à ce concours, de préparer ce qu'on appelle l'“auteur".»» (Mauss, 1925, p.482). 
assez nettement posées. On ne voit pas assez ou l'on va, quoi qu'on n'aille pas au hasard. Le lecteur n'est pas assez conduit. De même les différents tournants dans la [pensée ?] ne sont pas assez indiqués. Il faudrait se rendre bien compte de la thèse à établir, et en dessiner davantage le sectionnement ${ }^{4}$. $\gg$ C'est bien la construction de l'objet qui fait défaut. Les différents choix explicatifs faits par Mauss tout au long de son argumentation et les orientations, également variées, qu'ils donnent à son propos relèvent pour Durkheim d'un mouvement d'élargissement et de complexification du sujet, voire d'éclatement de la problématique, et non dans une tentative de réduction, ou encore de synthèse. L'accumulation d'exemples n'apprend rien, relance Durkheim, si ce n'est la pluralité des significations d'une notion. Un défaut qui, surtout, pourra être lu par les membres du jury comme le signe d’une contribution décousue.

Aussi, et pour chaque dissertation, le travail de correction est chirurgical. Durkheim supprime les mots en trop, remplace les tournures ambiguës, rappelle l'importance de la forme. Le moment s'y prête d’autant plus que la dissertation de philosophie est évaluée autant en termes de rigueur conceptuelle que d'unité entre la pensée et l'écriture. Outre ces commentaires en marge, Durkheim usera également de la correspondance pour amener Mauss à prendre conscience de la nécessité pour lui d'esquisser rapidement la marche à suivre, et ce de manière à rassurer les membres du jury et les auditeurs. Dans ses remarques, il ne cesse de rendre attentif son neveu - sans doute déjà trop «moderne» dans sa manière d’aborder certains sujets (Bert, 20ı16) - à la construction de ses phrases. Il s'agit d'éviter les négligences malheureuses, de supprimer les «enveloppements » et «les choses qui ne sont pas à leur place» (Durkheim, I894, p. 29). En I895, quelques semaines avant la date fatidique, Durkheim se fait encore plus précis et directif dans ses recommandations: «Dis tout ce que tu as à dire, mais mets-y le temps et les convenances nécessaires. Ne sois pas agressif [...]. Ne te crois pas obligé de tout dire à propos du sujet, dis une chose et tout ce qui est nécessaire pour établir cette chose, rien de plus en principe, de telle sorte que ta leçon dure cinquante minutes au moins mais pas plus de soixante.» (Durkheim, I895c, p. 38). 
Ce que cherche Durkheim, c'est d'enseigner la rigueur de l'École normale supérieure, là où, rappellera-t-il, se forme «l'élite de nos professeurs». Une école qui «donne le ton même à ceux qui n’en sortent pas» (Durkheim, I895b, p. 89). Bien plus que seulement «donner le ton », l'École normale génère chez ses élèves un éthos savant qui se manifeste par l'acquisition d'un style de pensée et de vie5. C'est d'ailleurs ce quáa voulu éviter à tout prix Mauss en choisissant, pour parfaire sa formation, le modèle universitaire de Bordeaux! Un moyen de cultiver sa liberté que Durkheim déplore (Durkheim, I898c, p. 499).

Bien que nous manquions d'un texte de Durkheim qui expose son attention pour l'écriture et la réécriture, son soin à l'expression des idées, notamment dans le cadre d'un raisonnement de type abstrait, se prête à deux sortes d'analyses. Premièrement, suivant les suggestions des tenants du paradigme herméneutique en ethnographie (Clifford Geertz et James Clifford), une idée serait d'enquêter sur le recours de Durkheim à des agencements argumentatifs particuliers, tel celui qui consiste à persuader son lecteur de prendre au sérieux ce qui est dit. De ce point de vue, le déploiement de cet intérêt constant pour la forme dénoterait un questionnement plus profond sur la place de l'énonciation. Formé à un moment où les lettres et les humanités classiques structurent l'érudition dominante, Durkheim «écrit» mais aussi pratique sa sociologie dans une autre culture, devenue essentiellement scientifique et technique ${ }^{6}$, qui suscite des réflexions renouvelées au sujet de l'exactitude, de l'objectivité et de la vérité. Comme le souligne Christophe Charles, qui s'est attaché aux parcours croisées de Durkheim, Henri Bergson et Jean Jaurès, il faudrait reprendre ce thème du rôle de la rhétorique en termes générationnels : «Imprégnés de cette rhétorique héritée des humanités classiques qu’on cultive dans les grands lycées parisiens, les classes préparatoires et l’École normale dans les années I870, ils en restent les héritiers reconnaissants mais critiques. Ils en usent quand c'est nécessaire, mais ils ont été aussi capables d’in-

5 Voir à ce sujet les remarques de Nicolas Adell (2016, p.61).

6 D'après Guillaume Carnino (2015, p. 123), entre 1895 et 1890 plus de 30 millions de francs seront investis dans les établissements privés pour financer la construction de laboratoires de «sciences appliquées au besoin de l'industrie [...], d'où une immense augmentation du nombre de travaux en "sciences appliquées" ». 
venter de nouvelles formes d'écriture et de s'ouvrir à de nouvelles techniques d’appréhension du monde. » (Charles, 20ı6, p. 426). Partant de là, il devient par exemple difficile de réduire les stratégies d'écriture utilisées par Durkheim au pur et simple bannissement de la subjectivité, comme cela serait certifié par l'emploi impératif et réitéré de sa part du «nous» académique.

Le second élargissement possible de cette question del'écriture pourrait consister à prolonger, du côté de la sociologie, la thèse éclairante de Vincent Debaene. Ce dernier a démontré, chez les ethnologues de la première moitié du $\mathrm{xx}^{\mathrm{e}}$ siècle, la coexistence d'une double écriture, littéraire et ethnologique au sens strict, qui ne relève pas uniquement des croisements de certains d'entre eux avec les écrivains surréalistes autour de la revue Documents. Cette coexistence de deux styles est de l'ordre d'un tropisme de la discipline, qui deviendra manifeste à partir de la création de l'Institut d'ethnologie de l'université de Paris en 1925 (Debaene, 20ı). Pourrait-on envisager une analyse semblable dans le cas de Durkheim? N'y-a-t-il pas dans son travail la trace d'une tension entre l'ambition d'un récit savant, objectif et neutre, fondé uniquement sur les faits et rejetant les belles apparences, et la prise en compte d'une subjectivité interprétative dans sa manière de restituer, mettre en scène ces mêmes faits (Lassave, 2002) ? Une tension qui pourrait désormais se penser sur la base du relevé des 505 emprunts du sociologue à la bibliothèque de Bordeaux (1889-1902) réalisé par Nicolas Sembel et Matthieu Béra (20I3). On trouve parmi ces emprunts des «distractions studieuses » qui nous rappellent que Durkheim a fait autre chose que de lire de la philosophie ou de la psychologie. La littérature fait également parti de ses préoccupations, en particulier le Journal et mémoires du marquis d'Argenson, les œuvres de Lucrèce et Plaute, Boileau, Goethe et Michelet.

\section{DE LA NÉCESSITÉ DE SAVOIR LIRE ENTRE LES LIGNES}

L’ambition épistémologique - même «scientiste» - de Durkheim est formellement affichée dans tous ses ouvrages. Dès les premières pages, il cherche à présenter sa méthode comme relevant d'un savoir voué à comprendre des phénomènes non immédiatement intelligibles. Son introduction au Suicide montre comment une telle approche - dès lors que le sociologue adopte 
une juste détermination de «l'ordre des faits», une définition correcte des termes du problème et la volonté de se prémunir contre «les rapprochements trompeurs ou les exclusions arbitraires »-peut finir par rendre une théorisation tout aussi solide que celle qui vaudrait dans les sciences dites exactes (Durkheim, I993 [I897], p. 2 et 6). C'est dans cette introduction, encore plus nettement que dans Les Règles, que Durkheim se sépare de la tradition érudite pour qui l'inventaire exhaustif des faits représentait l'alpha et l'oméga de tout travail scientifique. La tâche du sociologue est bien différente: choisir un nombre limité de facteurs explicatifs, en fonction de leur action sur l'ensemble de la société, et établir entre eux des corrélations signifiantes.

Cependant, peut-on saisir in concreto la manière dont Durkheim honore au quotidien son engagement? Pour cela, il est bon de se détourner autant que possible de ses textes majeurs et de ses formules stratégiques (traiter les faits sociaux comme des choses...). Durkheim est l'auteur d'une œuvre éparse, hétéroclite même, qui inclut des cours et des notes de cours, des interventions dans des débats, des comptes rendus et des correspondances. Autant de formats d'écritures qui composent ce que l'on pourrait appeler le «hors-texte » de l'œuvre. C’est dans ce horstexte qu'il produit, actualise des idées que l'on va retrouver dans ses livres et articles. C'est là, encore, que l'on peut voir comment il organise son activité, en particulier par le réemploi d’anciens matériaux ${ }^{7}$, et mesurer les contraintes auxquelles il est confronté. Cette focale prolonge un principe que Durkheim avait lui-même mis en place dans le but d'interroger l'enseignement scientifique: "partir des procédés réellement employés par les sciences, tels qu’elles les emploient, les décrire, les analyser, expliquer comment ils se sont formés, comment ils sont en rapport avec l'objet de chaque science, et c'est seulement ensuite qu'on pourrait, s'il y a lieu, remonter de proche en proche jusqu'à des procédés plus généraux dont les premiers ne sont que la diversification. » (Durkheim, I895a, p. 426). Deux outils «réellement employés » par Durkheim se signalent notamment.

7 II y aurait intérêt à forger un outil numérique capable de repérer dans le corpus durkheimien, les modalités et les séquences de son travail de pensée: par exemple l'usage de la littérature primaire et secondaire, le rapport entre l'ordre de la recherche et l'ordre d'exposition, ou encore le moment de la compilation et celui de l'invention. 
Le premier, familier des lecteurs des Règles qui y ont rencontré l'invitation à «échapper à l’empire des notions vulgaires » (Durkheim, I988 [1895], p. I15 et I27 $7^{\text {) }}$, consiste à s'éloigner du sens commun, tourner le dos à la connaissance ordinaire. On ne fait de la bonne sociologie que si on a le «vif sentiment de la diversité des choses et de leur complexité»(Durkheim et Fauconnet,1903, p. 154). Mettre de la distance entre un objet d'étude et soi, pratiquer le détachement, rappelle Durkheim dans L'Évolution pédagogique en France (1969 [1938], p. I54), devient un impératif méthodologique à la Renaissance déjà. C'est à cette époque que l'observateur des phénomènes sociaux, le futur sociologue mais aussi l'historien averti, commence à comprendre qu'il doit se méfier de leur apparence.

«Prudence » et «prudent» ce sont des mots que Durkheim aime également à employer. Sa seconde ficelle est que le sociologue doit se tenir sur ses gardes même face aux documents qu'il utilise (Durkheim, I908b, p. 200 ; I893, p. 242). Ce questionnement sur le document, sur sa fiabilité, s'accompagne d'un moment, plus ou moins long, où celui-ci doit être pensé «à nouveau pour son propre compte» et au besoin «remanié » pour se conformer aux autres pièces exploitées dans l'analyse (Durkheim, 1903a, p. 355). Pour cela, le sociologue doit savoir grouper ses données «d'une manière un peu artificielle» (Durkheim, 1905, p. I30). Question mordante pour la sociologie naissante car, et à la différence des sciences de la nature, il est impossible d'y employer l'expérimentation. Il faut donc en passer par des artifices méthodologiques permettant de «mettre le fait étudié dans des circonstances et sous des formes différentes afin que d'utiles comparaisons puissent être instituées» (Durkheim, I888, p. I3). Cette prudence sociologique doit aussi servir à contourner les «conjectures» risquées, les tissus de «pure imagination dont on peut parfois estimer l'ingéniosité, mais qui ne reposent sur aucune preuve positive» (Durkheim, 1904, p. I28). Ce n'est qu'en sélectionnant des faits à la fois observables et représentatifs qu'il est possible d'éviter «le tourbillonnement de faits» (Durkheim, 1903b, p. 94), c'est-à-dire la multiplication de documents-exemples peu probants, et les interprétations 
arbitraires (Durkheim, 1903a, p. 350 ; 1905, p. I3I) ${ }^{9}$. Après quoi, le sociologue doit encore savoir réfléchir à un plan et à un titre de sa contribution qui ne soit pas défectueux. Les deux doivent être pensés. Le titre, surtout, se doit d'être en accord avec le contenu. Dans le cas contraire, l'on pourrait croire à l'existence de «variations» dans la pensée de l’auteur (Durkheim, 1908c, p. 134).

\section{L'ORDINAIRE D'UNE DISCIPLINE VU PAR LA CORRESPONDANCE}

La réflexion que nous venons de mener sur les conditions pragmatiques du travail de Durkheim nous a permis, jusqu'ici, de revisiter autant ses textes intermédiaires que certaines de ses stratégies savantes. Elle doit encore être complétée par l'analyse de sa correspondance. C’est dans ses lettres, en effet, que Durkheim fait circuler d'autres éléments utiles à la compréhension de la façon dont il conçoit la discipline sociologique. Il est possible d'y mesurer certains progrès du point de vue de l'élaboration des concepts et des hypothèses ou de la terminologie adoptée. Des déterminations extra-scientifiques prennent parfois le dessus. Les questions théoriques se mélangent à des confidences sur des affaires personnelles et familiales, des soucis de carrière et de santé (intellectuelle et physique), des événements politiques. C’est dans cette correspondance que des confrontations se développent, animant pour un temps les discussions entre les collaborateurs de L'Année sociologique ou que les recrutements de futurs membres se discutent. Autant d'exemples qui nous rappellent que la sociologie durkheimienne, qu'elle soit prise sous l'angle de la conception, de l'édition ou de la diffusion, est d’abord une entreprise collective ${ }^{10}$.

9 «ll est pour le moins aussi important de bien choisir ceux [les faits] qu'il convient d'utiliser. II en est, en effet, qu'il faut résolument écarter, quelques instructifs qu'ils paraissent au premier abord.» (Durkheim, 1888, p. 17).

10 On se reportera à ce sujet à l'article de Muriel Lefebvre, Anne-Claire Jolivet et Sophie Dalle-Nazébi concernant l'écriture de la recherche: «L'écriture est toujours un acte coconstruit dans l'espace de l'“Autre". L'auteur n'est jamais seul d'un point de vue éditorial et s'inscrit dans une communauté épistémique qui construit un rapport un tant soit peu similaire au sien à leurs objets communs. » (Lefebvre, Jolivet et Dalle-Nazébi, 20 I5, p. 10). 
Dans ses lettres à Henri Hubert, on retrouve l'importance que Durkheim porte au style des textes à publier dans L'Année. Il ne s'agit jamais de simples questions de détail. Durkheim rassure Hubert quant à l'étendue de ses interventions, lui signalant, par exemple, qu'il n’a fait quajouter des points, des virgules, et «coup[é] les paragraphes» (Durkheim, 1900, p.502). Des changements qui tiennent le plus souvent au nombre de pages que l'éditeur, Felix Alcan, accorde à chaque volume de la revue. Certaines incises, des anecdotes, permettent de surprendre des «habitudes » plus singulières, comme la manière qu’a Durkheim de poser un obstacle et d'y répondre de la seule façon qu'il juge appropriée, à savoir en présentant les idées «par la pointe plutôt que par la poignée» (Durkheim, I897, p. 40I). Comme il l'écrira encore à Bouglé, c'est dans cet esprit qu'il décide d'intervenir dans les textes qui lui sont soumis, comme dans le cas de la traduction de l'article de Georg Simmel paru dans le premier volume (I898). Il recompose le titre et finalement l'article dans son entier, choisissant de redécouper les chapitres de manière ordonnée (Durkheim, I897b, p.408). Et, à propos des analyses d'ouvrages rédigées par Bouglé lui-même, qui occupent toute la première section du même volume, Durkheim lui fait remarquer que, en traçant «un canevas», ce qu'il n’a manifestement pas fait, Bouglé aurait pu «exprimer plus clairement» ce qu'il voulait dire (Durkheim, I897c, p. 4I0).

Les lettres de Durkheim à Hubert autorisent aussi un regard différent sur les modifications subies par la revue pendant sa courte existence (I898-1913). De manière générale, l'on a jusque-là expliqué ces changements par la mise en place, par Durkheim, d’une définition rigide de ce qui doit entrer dans le cadre de la réflexion sociologique - et donc de ce qui mérite ou non un compte rendu. On les a aussi interprétés comme des conséquences de l'acquisition, par Durkheim et son groupe, d'une position d'autorité savante, qu'il s'agissait pour eux de défendre face à d'autres acteurs, milieux ou institutions sociologiques concurrentes (Besnard, 2003, p. 283-297). D’autres aspects sont pourtant à considérer.

En premier lieu, l'on apprend de ces lettres que Durkheim a l'ambition de s'adresser à un lectorat mixte, «fait de gens qui, en partie, ne sont pas avec nous » (Durkheim, I898a, p.492), ce qui justifie aussi bien la recension par L’Année de travaux amateurs que la stratégie de Durkheim de publier dans des 
revues comme la Revue bleue ou la Revue de Paris. L'important c'est, au début, de faire sortir la sociologie d'un modèle qui ne vise qu'à rassembler des faits, sans les problématiser: «Non seulement [...] signaler les bons travaux mais [...] appeler la réflexion sur des points définis. » (Durkheim, I898b, p. 496). À partir du volume IV (I90I), le propos de Durkheim change. Il s'agit désormais d'avoir de l'influence. La revue doit compter dans les débats académiques : «Il faut que les érudits, tout en nous estimant, sachent que nous faisons autre chose qu'eux; que les sociologues, tout en nous craignant, sentent que nous faisons la même chose qu'eux, mais autrement. » (Durkheim, I9ora, p. 5I4). Aussi, outre les analyses d'ouvrages - qui lui coûtent un temps précieux (Durkheim, 1902, p. 524) - Durkheim mise sur les thèmes que la revue va traiter, des thèmes connus (la religion, tout d'abord) auxquels pourtant la sociologie apportera une lumière nouvelle. «Plus je réfléchis, plus je me persuade qu'il faut chercher à "enseigner" davantage, prendre le lecteur par la main et le mettre à notre point de vue.» (Durkheim, 190Ib, p. 516). La revue modifie sa périodicité en 1907 (vol. X). Elle paraîtra tous les trois ans et sans ses mémoires, du fait qu'Alcan sort la collection «Travaux de L’Année sociologique ». Pris par ses obligations universitaires, Durkheim en a désormais assez des analyses bibliographiques qu'il taxe d'interminables «travaux préparatoires ». Il faut «aboutir» martèle-t-il (Durkheim, 1906, p. 530). Ce qu'il vise c'est à marquer définitivement l'écart de la sociologie par rapport à d'autres disciplines, dont la philosophie et la psychologie (Durkheim, 1908a, p. 59). Cela implique une plus grande professionnalisation. Pour reprendre une idée foucaldienne, Durkheim s'engage dès lors vers une «disciplinarisation» du savoir sociologique $^{11}$. En 1908, Mauss expérimente à ses dépens cette accentuation de rigorisme. Durkheim refuse de publier la première version de sa préface aux Mélanges d'histoire des religions. Un texte, juge-t-il, «d'un ridicule achevé », et qui tranche autant par son ton que par un «personnalisme agaçant». Si un tel texte pourrait finir par desservir Mauss dans sa possible élection au Collège de France (il va se porter candidat en 1909), c'est surtout, pour Durkheim, la peur 
que ce soit l'ensemble des travaux de L'Année qui, par contrecoup, soit décrédibilisé par une telle publication (Durkheim, 1908d, p. 389).

L'œuvre de Durkheim, lorsqu'elle est réduite au caractère prétendument dogmatique et monolithique de sa pensée, présente encore de nombreuses difficultés pour le lecteur. L’aborder, comme nous suggérons de le faire ici, par la focale de l'anthropologie des pratiques savantes - et donc en essayant de déceler les étapes, les interrogations, les faux-pas et les soucis qui ont accompagné son élaboration - ne peut que contribuer à en enrichir la connaissance et peut-être la modernité.

\section{BIBLIOGRAPHIE}

ADELL N., 2016, «Ouverture: la vie savante. Perspectives morphologiques», in N. Adell et J. Lamy (dir.), 2016, Ce que la science fait à la vie, Paris, CTHS, p. 21-77.

BACIOCCHI S. et MARIOT N., 2015, «Robert Hertz, été 1912》, in R. Hertz, Sociologie religieuse et anthropologie. Deux enquêtes de terrain, 1912-1915, Paris, PUF, p.7-4I.

BECKER H. S., 2002 (1988), Les Ficelles du métier. Comment conduire sa recherche en sciences sociales, Paris, La Découverte.

BERT J.-F., 2012, L'Atelier de Marcel Mauss. Un anthropologue paradoxal, Paris, Éditions du CNRS.

-, 2016, «Marcel Mauss, un (in)disciple de Durkheim? », in ADELL et LAMY, 2016, p. 307-321.

BESNARD Ph., 2003, Études durkheimiennes, Genève, Droz.

CARNINO G., 2015, L'Invention de la science, Paris, Éditions du Seuil.

CHARLES Ch., 2016, «Bergson, Durkheim, Jaurès», in Ch. Charles et L. Jeanpierre (dir.), La Vie intellectuelle en France, Paris, Éditions du Seuil, vol. I, p.424-427.

DEBAENE V., 20I0, L'Adieu au voyage: l'ethnologie française entre science et littérature, Paris, Gallimard.

DURKHEIM É., 1888, «Introduction à la sociologie de la famille », Annales de la Faculté des lettres de Bordeaux, I0, [p.257-28I]. Repris in DURKHEIM, 1975, vol.3, p.9-34. 
-, 1893, «C. r. de G. Richard, Essai sur l'origine de l'idée de droit (1892)», Revue philosophique, 35, [p. 290-296]. Repris comme «Origine de l'idée de droit», in DURKHEIM, 1975, vol. I, p. 233-240.

-, 1894, «Lettre à M. Mauss de février ou mars», in DURKHEIM, 1998, p. 28-31.

-, 1895a, «L'enseignement philosophique et l'agrégation de philosophie», Revue philosophique, 39, [p. 121-147]. Repris in DURKHEIM, 1975, vol. 3, p. 403-436.

-, 1895b, «Lo stato attuale degli studi sociologici in Francia», La Riforma sociale, 3, [p. 607-622, 691-707]. Repris in DURKHEIM, 1975, vol I, p.73-108.

-, 1895c, «Lettre à M. Mauss de juillet», in DURKHEIM, 1998, p. 37-38.

-, 1897a, «Lettre à C. Bouglé du 6 juillet», in DURKHEIM, 1975, vol.2, p. 399-402.

-, 1897b, «Lettre à C. Bouglé du 18 septembre», in DURKHEIM, 1975, vol.2, p. 407-408.

-, 1897c, «Lettre à C. Bouglé du 27 septembre», in DURKHEIM, 1975, vol. 2, p. 409-4I2.

-, 1898a, «Lettre à H. Hubert du 30 mars », in DURKHEIM, 1987, p. 492-494.

-, 1898b, «Lettre à H. Hubert du 28 avril », in DURKHEIM, 1987, p. 496-497.

-, I898c, «Lettre à H. Hubert de novembre ou décembre», in DURKHEIM, 1987, p. 498-499.

-, 1899a, «Contribution à l'“Enquête sur l'introduction de la sociologie dans l'enseignement secondaire"”, Revue internationale de sociologie, 7, [p. 679]. Repris comme «Remarque sur l'enseignement de la sociologie», in DURKHEIM, 1975, vol. I, p. 51-52.

-, 1899b, «Lettre à C. Bouglé du 9 mai », in DURKHEIM, 1975, vol. 2, p. 430-432.

-, 1900, «Lettre à H. Hubert du 16 janvier», in DURKHEIM, 1987, p. 498-499.

-, 190 la, «Lettre à H. Hubert du 14 janvier», in DURKHEIM, 1987, p. 5I2-5I4.

-, 190 Ib, «Lettre à H. Hubert du 6 février», in DURKHEIM, 1987, p. 515-5I7.

-, 1902, «Lettre à H. Hubert du 10 mars», in DURKHEIM, 1987, p. 524-525.

-, 1903a, «Intervention à la soutenance de thèse de Ch. Ribéry, Essai sur les caractères», Revue de métaphysique et de morale, II, supp. au n. de juillet, [p. 21-22]. Repris comme «Débat sur la classification des caractères et la pédagogie», in DURKHEIM, 1975, vol. 3, p.349-35I. 
-, 1903b, «C. r. de E. Crawley, The Mystic Rose. A Study of Primitive Marriage (1902)», L'Année sociologique, 6, [p. 352-358]. Repris comme «Mariage et sexualité primitifs selon Crawley», in DURKHEIM, 1975, vol.3, p.94-101.

-, 1904, «Réponse à Mr Lang», Folklore, 15, [p. 215-216]. Repris comme«À propos du totémisme. Réponse à Lang», in DURKHEIM, 1975, vol. 2, p. 128-129.

-, 1905, «Intervention au débat à la soutenance de thèse de Louis-Germain Lévy. Résumé établi par Paul Fontana», Revue de philosophie, 5, [p. 486-489]. Repris comme «Le problème de la solidarité familiale et du totémisme chez les Hébreux», in DURKHEIM, 1975, vol.2, p. 130-133.

-, 1906, «Lettre à H. Hubert de mai-juin», in DURKHEIM, 1987, p.530-531.

-, 1907, «Cours d'Émile Durkheim à la Sorbonne», Revue de philosophie, 7, [p.528-539]. Repris comme «Cours sur les origines de la vie religieuse», in DURKHEIM, 1975, vol.2, p.65-122.

-, 1908a, «Contribution à une "Enquête sur la sociologie"», Les Documents du progrès, 2, [p. 131-133]. Repris comme «Remarque sur la méthode en sociologie», in DURKHEIM, 1975, vol. I, p.58-61.

-, 1908b, «Intervention à la discussion sur "L'inconnu et l'inconscient en histoire" à la séance du 28 mai 1908 de la Société française de philosophie», Bulletin de la Société française de philosophie, 8, [p. 229-247]. Repris comme «Débat sur l'explication en histoire et en sociologie», in DURKHEIM, 1975, vol. I, p. 199-217.

-, 1908c, «Intervention à la soutenance de thèse de Revault d'Allones, Psychologie d'une religion», Revue de métaphysique et de morale, 16, supp.au n. de mars, [p.31-32]. Repris comme «Débat sur la psychosociologie du prophétisme», in DURKHEIM, 1975, vol.2, p. 134-136.

-, 1908d, «Lettre à Marcel Mauss de novembre», in DURKHEIM, 1998, p. 389-390

-, 1915, «La sociologie», in Ministère de l'Instruction publique et des Beaux-Arts, La Science française, Paris, Larousse, I, [p. 39-49]. Repris in DURKHEIM, 1975, vol. I, p. $109-118$.

-, 1969 (1938), L'Évolution pédagogique en France, Paris, PUF.

-, 1975, Textes, Paris, Les Éditions de Minuit, 3 vol.

-, 1987, «Lettres de Émile Durkheim à Henri Hubert », Revue française de sociologie, 28-3, p. 483-534. 
-, 1988 (1895), Les Règles de la méthode sociologique, Paris, Flammarion.

-, 1993 (1897), Le Suicide. Étude de sociologie, Paris, PUF.

-, 1998, Lettres à Marcel Mauss, Paris, PUF.

- et P. FAUCONNET, 1903, «Sociologie et sciences sociales», Revue philosophique, 55, [p.465-497]. Repris in DURKHEIM, 1975, vol. I, p. I2I-165.

FABRE D. (dir.), 1997, Par écrit, ethnologie des écritures quotidiennes, Paris, Éditions de la MSH.

FOUCAULT M., 1997, «ll faut défendre la société». Cours au Collège de France, 1975-1976, Paris, EHESS-Gallimard-Éditions du Seuil.

JACOB Ch. (dir.), 20II, Lieux de savoir - 2. Les mains de l'intellect, Paris, Albin Michel.

LASSAVE P., 2002, Sciences sociales et littérature, Paris, PUF.

LEFEBVRE M., JOLIVET A.-Cl. et DALLE-NAZEBI S., 20I5, «Les écritures ordinaires des chercheurs», in J.-Fr. Bert et M. J. Ratcliff (dir.), Frontières d'archives: recherches, mémoires, savoirs, Paris, Éditions des archives contemporaines, p. I-13.

MAUSS M., 1925, «In memoriam. L'œuvre inédite de Durkheim et de ses collaborateurs», L'Année sociologique, n. s., I, [p.7-29]. Repris in Id., Euvres, Paris, Éditions de Minuit, 1968-1969, vol. 3, p. 473-499.

SEMBEL N., 20I3, «La liste des emprunts de Durkheim à la bibliothèque universitaire de Bordeaux: une "imagination méthodologique" en acte», Durkheimian Studies / Études Durkheimiennes, 19 (n. s.), p.5-48.

TOPALOV Ch., 2012, «Maurice Halbwachs: une experience américaine », in M. Halbwachs, Écrits d'Amérique, Paris, Éditions de l'EHESS, p. II-77. 